\title{
Facile synthesis of a comb-like mannohexaose: a trimer of the disaccharide repeating unit of the cell-wall mannans of Aphanoascus mephitatus and related species
}

\author{
Linsen Heng, Jun Ning, Fanzuo Kong* \\ Research Center for Eco-Environmental Sciences, Chinese Academy of Sciences, PO Box 2871, \\ Beijing 100085, People's Republic of China
}

Received 27 November 2000; accepted 16 February 2001

\begin{abstract}
An efficient method for the preparation of a comb-like mannohexaose having $\alpha-(1 \rightarrow 6)$ and $\alpha-(1 \rightarrow 2)$ linkages has been described using 6- $O$-acetyl-2- $O$-benzoyl-3,4-di- $O$-benzyl- $\alpha$-D-mannopyranosyl trichloroacetimidate as the key glycosyl donor in an 'inverse Schmidt' procedure. (C) 2001 Elsevier Science Ltd. All rights reserved.
\end{abstract}

Keywords: Comb-like oligosaccharide; Synthesis; Mannose

\section{Introduction}

Mannans are constituents of many fungal cell-walls. They are considered to have important physiological functions. Besides physical protection of the cell-wall, they may serve to anchor such enzymes as invertase and acid
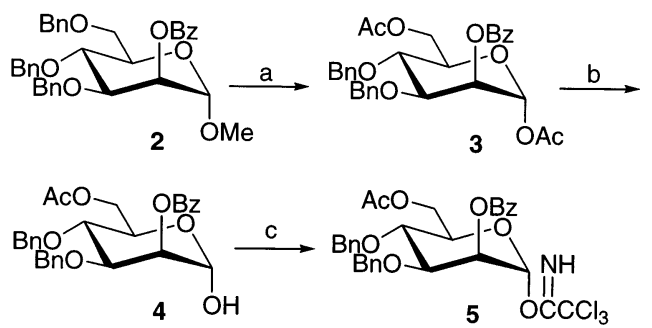

Scheme 1. Reagents and conditions: (a) 6:1:0.05 (v/v) AcOH$\mathrm{Ac}_{2} \mathrm{O}-\mathrm{H}_{2} \mathrm{SO}_{4}, \mathrm{rt}, 16 \mathrm{~h}, 90 \%$; (b) anhyd $\mathrm{Et}_{2} \mathrm{O}$ satd with dry $\mathrm{NH}_{3}, \mathrm{rt}, 24 \mathrm{~h}, 97 \%$; (c) $\mathrm{CCl}_{3} \mathrm{CN}$ (1.2 equiv), DBU (0.2 equiv), $0{ }^{\circ} \mathrm{C}, 2 \mathrm{~h}, 92 \%$.

* Corresponding author. Tel.: + 86-10-62936613; fax: + 86-10-62923563.

E-mail address: fzkong@mail.rcees.ac.cn (F. Kong). phosphatase to the cell-wall, ${ }^{1}$ and they may participate in cell-cell recognition as well as in the adhesion of the microorganism to host cells.

In 1993 Jiménez-Barbero and co-workers ${ }^{2}$ investigated the structures of cell-wall mannans isolated from Aphanoascus mephitatus, Aphanoascus fulvescens, Aphanoascus verrucosus, and Aphanoascus reticulisporus, and found that they invariably consist of a relatively simple comb-like structure of a disaccharide repeating block $\{\rightarrow 6\}$-[ $\alpha$-Man $p-(1 \rightarrow 2)]-\alpha-$ Man $p-(1 \rightarrow)$. It would be helpful to synthesize its fragments for the elucidation of the biological functions of the cell-wall polysaccharides. In a previous communication, ${ }^{3}$ we described an efficient method to construct a mannooligosaccharide having $\alpha-(1 \rightarrow 6)$ and $\alpha$ $(1 \rightarrow 2)$ linkages. In this paper, as a part of our continuing effort to develop new synthetic approaches directed toward oligosaccharide fragments of fungal cell-wall mannans, we report the synthesis of a comb-like haxasac- 


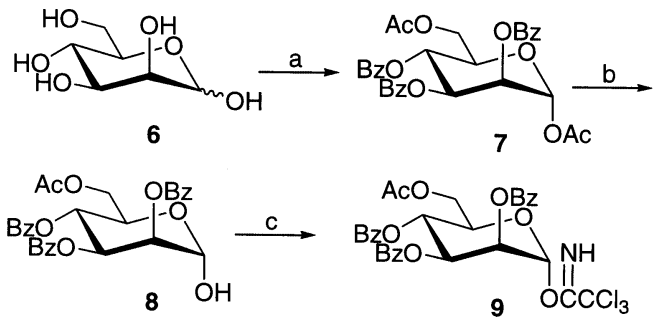

Scheme 2. Reagents and conditions: (a) (i) $\mathrm{TrCl}$ (1.2 equiv), pyridine, $50{ }^{\circ} \mathrm{C}, 32 \mathrm{~h}$; (ii) $\mathrm{BzCl}$ (4.4 equiv), $40^{\circ} \mathrm{C}, 24 \mathrm{~h}$; (iii) 1:1:0.6:0.175 (v/v) $\mathrm{CH}_{2} \mathrm{Cl}_{2}-\mathrm{HOAc}-\mathrm{Ac}_{2} \mathrm{O}-\mathrm{H}_{2} \mathrm{SO}_{4}, \mathrm{rt}, 20 \mathrm{~h}$, $71.3 \%$. (b) $\mathrm{THF}, \mathrm{BnNH}_{2}$ (4.2 equiv), rt, $24 \mathrm{~h}, 86.2 \%$. (c) $\mathrm{CCl}_{3} \mathrm{CN}$ (2.8 equiv), DBU (0.22 equiv), $0{ }^{\circ} \mathrm{C}, 2 \mathrm{~h}, 88.1 \%$.

charide $\mathbf{1}$, which is a trimer of the disaccharide repeating unit of mannans isolated from $A$. mephitatus and related species.

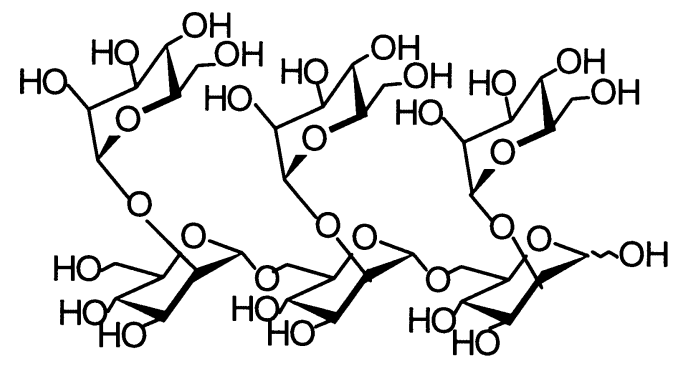

1

\section{Results and discussion}

First we synthesized the synthons 5 and $\mathbf{9}$. Thus, methyl 2-O-benzoyl-3,4,6-tri- $O$-benzyl$\alpha$-D-mannopyranoside (2) was prepared in $65 \%$ overall yield according to the literature ${ }^{4}$ using D-mannose as the starting material. Selective acetolysis of $\mathbf{2}$ using 6:1:0.05 HOAc$\mathrm{Ac}_{2} \mathrm{O}-\mathrm{H}_{2} \mathrm{SO}_{4}{ }^{5}$ gave the corresponding diacetate 3 (Scheme 1). Compound 4 was obtained in nearly quantitative yield by amonolysis of $\mathbf{3}$ in anhydrous ether saturated with dry $\mathrm{NH}_{3}$ according to Lemieux and Howard. ${ }^{6}$ Subsequent reaction of 4 with $\mathrm{CCl}_{3} \mathrm{CN}-\mathrm{DBU}$ in $\mathrm{CH}_{2} \mathrm{Cl}_{2}$ afforded the key glycosyl donor 5. Tritylation of the mannose (6), ${ }^{7}$ followed by benzoylation in one pot, gave the 1,2,3,4-tetra- $O$-benzoyl-6- $O$-trityl-Dmannopyranose (Scheme 2), selective acetolysis of which using 1:1:0.6:0.175 $\mathrm{CH}_{2} \mathrm{Cl}_{2}-$ $\mathrm{AcOH}-\mathrm{Ac}_{2} \mathrm{O}-\mathrm{H}_{2} \mathrm{SO}_{4}$ afforded the corresponding 1,6-diacetate 7 in $85 \%$ yield (three steps). The diacetate 7 was selectively deacety- lated in high yield at the anomeric position with benzylamine in THF to the corresponding 6- $O$-acetyl-2,3,4-tri- $O$-benzoyl-D-mannopyranose (8). Subsequent reaction of $\mathbf{8}$ with $\mathrm{CCl}_{3} \mathrm{CN}-\mathrm{DBU}$ in $\mathrm{CH}_{2} \mathrm{Cl}_{2}$ afforded another glycosyl donor 9.

With the synthons 5 and 9 in hand, construction of the target compound was readily carried out. As shown in Scheme 3, allyl 6-Oacetyl - 2 - $O$ - benzoyl - 3,4 - di - $O$ - benzyl - $\alpha$ - Dmannopyranoside (10) was prepared by the Helferich reaction ${ }^{8}$ using $\mathbf{3}$ as the glycosyl donor and allyl alcohol as the acceptor. Selective removal of the acetyl group of $\mathbf{1 0}$ using a $0.5 \%$ methanolic $\mathrm{HCl}$ quantitatively gave the glycosyl acceptor 11. The disaccharide 12 was prepared using the 'inverse Schmidt' strategy. ${ }^{9}$ Thus the glycosyl acceptor $\mathbf{1 1}$ and the catalyst TMSOTf were mixed first in dry $\mathrm{CH}_{2} \mathrm{Cl}_{2}$, and after stirring for $15 \mathrm{~min}$, the glycosyl donor $\mathbf{5}$ was added dropwise within $30 \mathrm{~min}$. Selective removal of the acetyl group of $\mathbf{1 2}$ gave the glycosyl acceptor 13, 'inverse Schmidt' coupling of which with 2-O-acetyl-3,4,6-tri- $O$ benzyl- $\alpha$-D-mannopyranosyl trichloro-acetimidate $^{10}$ afforded the trisaccharide $\mathbf{1 4}$ in $85 \%$ yield. The ${ }^{1} \mathrm{H}$ NMR spectrum of 14 showed one acetyl signal ( $\delta$ 2.14), one allyl methine signal (5.87) and three downfield $\mathrm{H}-2$ signals ( $\delta 5.78,5.67$, and 5.52), characteristic of the structure of the trisaccharide 14. Removal of the acetyl and benzoyl groups of 14 with $\mathrm{NH}_{3}$ in $\mathrm{MeOH}$ quantitatively gave the glycosyl acceptor 15 having free 2-OH, 2'-OH, and $2^{\prime \prime}$ $\mathrm{OH}$ groups. The fully protected comb-like hexasaccharide 16 was smoothly obtained by coupling of 15 with 6-O-acetyl-2,3,4-tri- $O$ benzoyl- $\alpha$-D-mannopyranosyl trichloroacetimidate (9). The ${ }^{1} \mathrm{H}$ and ${ }^{13} \mathrm{C}$ NMR data of $\mathbf{1 6}$ contained structural information, i.e., three acetyl signals $(\delta 1.91,1.93$, and 1.98) and one allyl methine signal ( $\delta$ 5.93), and six anomeric carbon signals $(\delta 99.6,99.4,99.3,99.2 .99 .1$, and 98.3). The use of 6-O-acetyl-2,3,4-tri- $O$ benzoyl- $\alpha$-D-mannopyranosyl trichloroacetimidate (9) as the glycosyl donor afforded three hydroxy groups at C-6 for potential further transformation. Deprotection of $\mathbf{1 6}$ gave the title mannohexaose 1 .

In summary, we have successfully developed a highly efficient strategy for the preparation 
of a comb-like mannohexaose containing $\alpha$ $(1 \rightarrow 6)$ and $\alpha-(1 \rightarrow 2)$ linkages. It is anticipated that octa- and higher comb-like oligosaccharides can be synthesized by this strategy.

\section{Experimental}

General methods.-Optical rotations were determined at $25^{\circ} \mathrm{C}$ with a Perkin-Elmer model 241-MC automatic polarimeter. Melting points were determined with a 'Mel-Temp' apparatus. ${ }^{1} \mathrm{H}$ NMR spectra were recorded in $\mathrm{CDCl}_{3}$ with Bruker ARX 400 spectrometers. Chemical shifts are given in parts per million (ppm) downfield from internal $\mathrm{Me}_{4} \mathrm{Si}$. Mass spectra were recorded with a JMS-D300S mass spectrometer using a sample probe. Thin-layer chromatography (TLC) was performed on Silica Gel $\mathrm{HF}_{254}$ (E. Merck) with detection by charring with $30 \%$ (v/v) $\mathrm{H}_{2} \mathrm{SO}_{4}$ in $\mathrm{MeOH}$ or in some cases by UV detection. Column chromatography utilized silica gel with EtOAc-petroleum ether $\left(60-90^{\circ} \mathrm{C}\right)$ as the eluent. Solutions were concentrated at $<$ $60{ }^{\circ} \mathrm{C}$ under diminished pressure.

1,6-Di-O-acetyl-2-O-benzoyl-3,4-di-O-ben$z y l-\alpha$-D-mannopyranose (3). - A solution of compound $2^{4}(6 \mathrm{~g}, 10.55 \mathrm{mmol})$ in $\mathrm{AcOH}(36$ $\mathrm{mL})$ and $\mathrm{Ac}_{2} \mathrm{O}(6 \mathrm{~mL})$ cooled to $0{ }^{\circ} \mathrm{C}$ in an ice bath, and $\mathrm{H}_{2} \mathrm{SO}_{4}(0.3 \mathrm{~mL})$ was added dropwise over $10 \mathrm{~min}$. The ice bath was removed, and the reaction was allowed to continue for $16 \mathrm{~h}$ at $\mathrm{rt}$, then poured into ice water $(100$ $\mathrm{mL}$ ). Stirring was continued for an additional $15 \mathrm{~min}$, at the end of which time it was extracted with $\mathrm{CHCl}_{3}(3 \times 30 \mathrm{~mL})$. The combined $\mathrm{CHCl}_{3}$ extracts were washed with $10 \%$ aq $\mathrm{NaHCO}_{3}(3 \times 60 \mathrm{~mL})$, dried over $\mathrm{Na}_{2} \mathrm{SO}_{4}$, and concentrated to a syrup. The crude product was then subjected to column chromatography with $3: 1$ petroleum ether-EtOAc as the eluent. Compound $\mathbf{3}$ was obtained as crystals $\left(5.21 \mathrm{~g}, 90 \%\right.$,); mp $102-104{ }^{\circ} \mathrm{C} ;[\alpha]_{\mathrm{D}}$ $+3.2^{\circ}\left(c .1 .4, \mathrm{CHCl}_{3}\right) ;{ }^{1} \mathrm{H}$ NMR $\left(\mathrm{CDCl}_{3}, 400\right.$ $\mathrm{MHz}): \delta 8.20-7.20(\mathrm{~m}, 15 \mathrm{H}, 3 \mathrm{Ph} H), 6.20(\mathrm{~d}$, $\left.1 \mathrm{H}, J_{1,2} 2.1 \mathrm{~Hz}, \mathrm{H}-1\right), 5.62\left(\mathrm{dd}, 1 \mathrm{H}, J_{1,2} 2.1\right.$, $\left.J_{2,3} 2.9 \mathrm{~Hz}, \mathrm{H}-2\right), 4.89,4.60(\mathrm{ABq}, 2 \mathrm{H}, J 10.7$
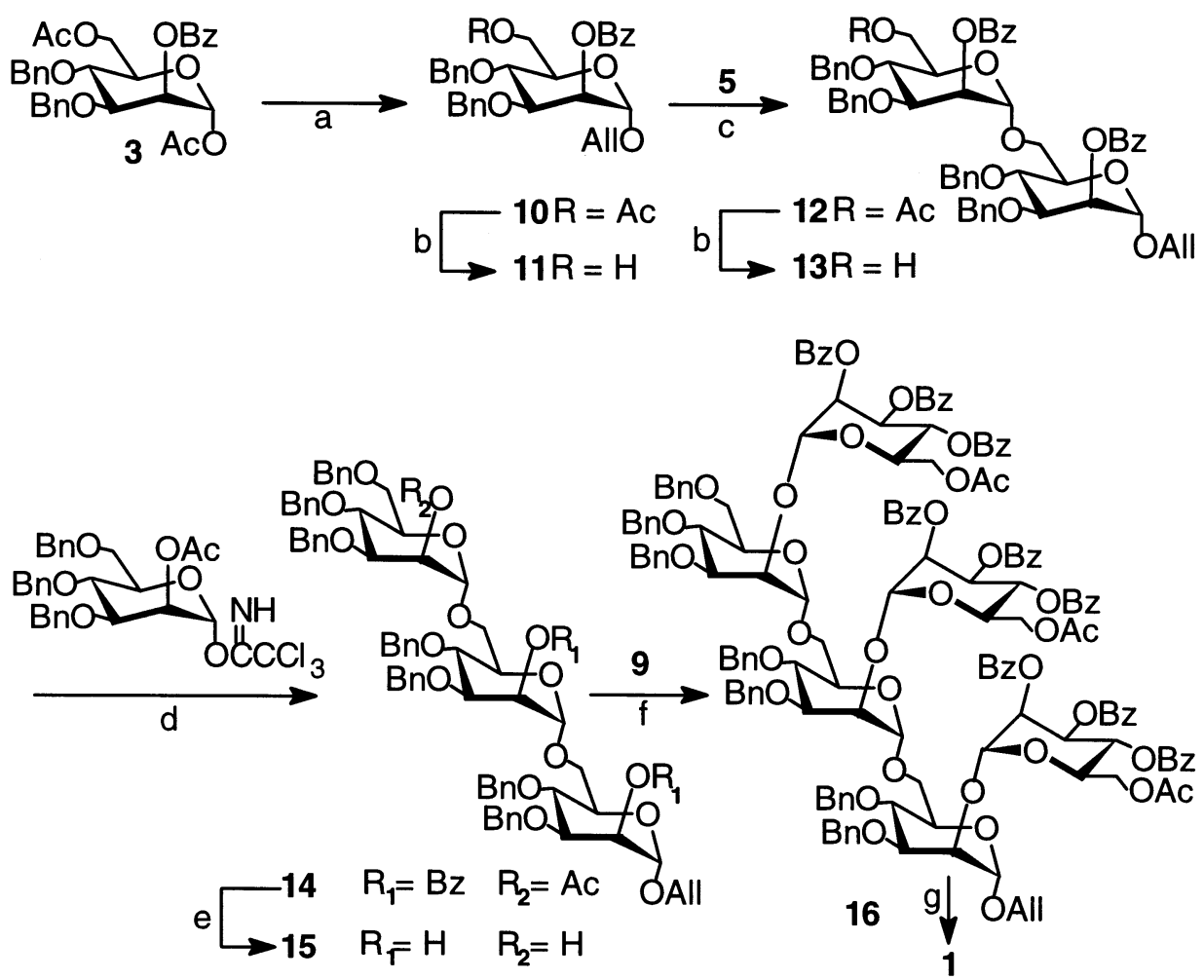

Scheme 3. Reagents and conditions: (a) allyl alcohol (1.9 equiv), TMSOTf (0.24 equiv), $\mathrm{CH}_{2} \mathrm{Cl}_{2}, \mathrm{rt}, 1 \mathrm{~h}, 88 \%$. (b) $\mathrm{MeOH}-0.5 \%$ $\mathrm{HCl}, \mathrm{rt}, 14-18 \mathrm{~h}, 95.6-98.1 \%$. (c) 5 (1.4 equiv), $\mathrm{CH}_{2} \mathrm{Cl}_{2}$, TMSOTf (0.1 equiv), rt, $3 \mathrm{~h}, 86.5 \%$. (d) 2-O-Acetyl-3,4,6-tri- $O$-benzyl- $\alpha$ D-mannopyranosyl trichloroacetimidate (1.5 equiv), $\mathrm{CH}_{2} \mathrm{Cl}_{2}$, TMSOTf $\left(0.1\right.$ equiv), rt, $3 \mathrm{~h}, 85.7 \%$. (e) $\mathrm{MeOH}$ satd with dry $\mathrm{NH}_{3}$, rt, 72 h, 97\%. (f) 9 (6.0 equiv), $\mathrm{CH}_{2} \mathrm{Cl}_{2}$, TMSOTf (0.2 equiv), rt, 3 h, 75.8\%. (g) (i) $\mathrm{PdCl}_{2}, \mathrm{MeOH}, \mathrm{rt}, 8$ h; (ii) $\mathrm{MeOH}$ satd with dry $\mathrm{NH}_{3}$; (iii) $\mathrm{H}_{2}, \mathrm{Pd}(\mathrm{OH})_{2} / \mathrm{C} 20 \%, \mathrm{MeOH}, \mathrm{rt}, 24 \mathrm{~h}, 61.8 \%$. 
$\left.\mathrm{Hz}, \mathrm{PhCH}_{2}\right), 4.83,4.61(\mathrm{ABq}, 2 \mathrm{H}, J 11.9 \mathrm{~Hz}$, $\left.\mathrm{PhCH}_{2}\right), 4.34$ (m, $\left.2 \mathrm{H}, \mathrm{H}-6,6^{\prime}\right), 4.11$ (m, $1 \mathrm{H}$, $\mathrm{H}-5), 3.96$ (m, $2 \mathrm{H}, \mathrm{H}-3,4), 2.10,2.06$ (2 s, 6 $\mathrm{H}, 2 \mathrm{COCH}_{3}$ ). Anal. Calcd for $\mathrm{C}_{31} \mathrm{H}_{32} \mathrm{O}_{9}: \mathrm{C}$, 67.87; H, 5.88. Found: C, 67.95; H, 5.79.

6-O-Acetyl-2-O-benzoyl-3,4-di-O-benzyl- $\alpha$ D-mannopyranose (4). - A solution of compound 3 (3 g, $5.47 \mathrm{mmol})$ in anhyd ether (100 $\mathrm{mL})$ saturated with dry $\mathrm{NH}_{3}$ was stirred for 24 $h$ at $\mathrm{rt}$. TLC (2:1 petroleum ether-EtOAc) indicated that the reaction was complete. The solution was concentrated, and purification of the crude product by flash-column chromatography on silica gel (2:1 petroleum etherEtOAc) gave compound $\mathbf{4}$ as crystals $(2.69 \mathrm{~g}$, $97 \%$, $; \operatorname{mp~} 108-110{ }^{\circ} \mathrm{C} ;[\alpha]_{\mathrm{D}}+6.9^{\circ}$ (c 2.1, $\left.\mathrm{CHCl}_{3}\right) ;{ }^{1} \mathrm{H}$ NMR $\left(\mathrm{CDCl}_{3}, 400 \mathrm{MHz}\right): \delta$ 8.07-7.23 (m, $15 \mathrm{H}, 3 \mathrm{PhH}), 5.60(\mathrm{dd}, 1 \mathrm{H}$, $\left.J_{1,2} 1.8, J_{3,2} 2.8 \mathrm{~Hz}, \mathrm{H}-2\right), 5.31\left(\mathrm{~d}, 1 \mathrm{H}, J_{2,1} 1.8\right.$ $\mathrm{Hz}, \mathrm{H}-1), 4.89,4.59$ (ABq, $2 \mathrm{H}, J 10.9 \mathrm{~Hz}$, $\left.\mathrm{PhCH}_{2}\right), 4.79,4.58(\mathrm{ABq}, 2 \mathrm{H}, J 11.3 \mathrm{~Hz}$, $\left.\mathrm{PhCH}_{2}\right), 4.38\left(\mathrm{dd}, 1 \mathrm{H}, J_{6 \mathrm{~b}, 6 \mathrm{a}} 11.9, J_{5,6 \mathrm{a}} 2.2 \mathrm{~Hz}\right.$, H-6a), 4.27 (dd, $1 \mathrm{H}, J_{6 \mathrm{a}, 6 \mathrm{~b}} 11.9, J_{5,6 \mathrm{~b}} 4.4 \mathrm{~Hz}$, $\mathrm{H}-6 \mathrm{~b}), 4.18$ (dd, $1 \mathrm{H}, J_{2,3} 2.8, J_{4,3} 9.1 \mathrm{~Hz}, \mathrm{H}-3$ ), $4.12\left(\mathrm{~m}, 1 \mathrm{H}, J_{6 \mathrm{a}, 5} 2.2, J_{6 \mathrm{~b}, 5} 4.4, J_{4,5} 9.1 \mathrm{~Hz}\right.$, $\mathrm{H}-5), 3.90\left(\mathrm{t}, 1 \mathrm{H}, J_{3,4}=J_{5,4}=9.1 \mathrm{~Hz}, \mathrm{H}-4\right)$, 2.04 (s, $\left.3 \mathrm{H}, \mathrm{COCH}_{3}\right)$. Anal. Calcd for $\mathrm{C}_{29} \mathrm{H}_{30} \mathrm{O}_{8}$ : C, 68.76; H, 5.97. Found: C, 68.61; $\mathrm{H}, 5.92$.

6-O-Acetyl-2-O-benzoyl-3,4-di-O-benzyl- $\alpha$ D-mannopyranosyl trichloroacetimidate (5).A mixture of 4 (4.5 g, $8.88 \mathrm{mmol}), \mathrm{CCl}_{3} \mathrm{CN}$ (1.05 mL, $10.5 \mathrm{mmol}$ ), and 1,8-diazabicyclo[5,4,0] undecene (DBU) $\quad(0.27 \mathrm{~mL}, 1.8$ mmol) in dry $\mathrm{CH}_{2} \mathrm{Cl}_{2}(30 \mathrm{~mL})$ was stirred under $\mathrm{N}_{2}$ for $2 \mathrm{~h}$ at $0{ }^{\circ} \mathrm{C}$ and then concentrated in vacuo. The residue was purified by flash chromatography (3:1 petroleum etherEtOAc) to give $5(5.32 \mathrm{~g}, 92 \%)$ as crystals; $\mathrm{mp}$ $91-93{ }^{\circ} \mathrm{C} ;[\alpha]_{\mathrm{D}}+16.9^{\circ}$ (c 2.6, $\mathrm{CHCl}_{3}$ ); ${ }^{1} \mathrm{H}$ NMR $\left(\mathrm{CDCl}_{3}, 400 \mathrm{MHz}\right): \delta 8.72(\mathrm{~s}, 1 \mathrm{H}$, $\left.\mathrm{OC}(\mathrm{NH}) \mathrm{CCl}_{3}\right), 8.10-7.10(\mathrm{~m}, 15 \mathrm{H}, 3 \mathrm{PhH})$, $6.36\left(\mathrm{~d}, 1 \mathrm{H}, J_{1,2} 2.1 \mathrm{~Hz}, \mathrm{H}-1\right), 5.73(\mathrm{t}, 1 \mathrm{H}$, $\left.J_{1,2}=J_{2,3}=2.1 \mathrm{~Hz}, \mathrm{H}-2\right), 4.90,4.64(\mathrm{ABq}, 2$ $\left.\mathrm{H}, J 10.8 \mathrm{~Hz}, \mathrm{PhCH}_{2}\right), 4.82,4.61(\mathrm{ABq}, 2 \mathrm{H}$, $\left.J 11.3 \mathrm{~Hz}, \mathrm{PhCH}_{2}\right), 4.35\left(\mathrm{dd}, 1 \mathrm{H}, J_{6,6^{\prime}} 11.9\right.$, $\left.J_{5,6} 1.7 \mathrm{~Hz}, \mathrm{H}-6\right), 4.30\left(\mathrm{dd}, 1 \mathrm{H}, J_{6,6^{\prime}} 11.9, J_{5,6^{\prime}}\right.$ $\left.3.4 \mathrm{~Hz}, \mathrm{H}-6^{\prime}\right), 4.17$ (dd, $1 \mathrm{H}, J_{2,3} 2.1, J_{3,4} 8.7$ $\mathrm{Hz}, \mathrm{H}-3), 4.1\left(\mathrm{t}, 1 \mathrm{H}, J_{3,4}=J_{4,5}=8.7 \mathrm{~Hz}\right.$, $\mathrm{H}-4), 4.02\left(\mathrm{~m}, 1 \mathrm{H}, J_{5,6} 1.7, J_{5,6}, 3.4, J_{4,5} 8.7\right.$ $\mathrm{Hz}, \mathrm{H}-5), 2.03$ (s, $3 \mathrm{H}, \mathrm{COCH}_{3}$ ). Anal. Calcd for $\mathrm{C}_{31} \mathrm{H}_{30} \mathrm{Cl}_{3} \mathrm{NO}_{8}$ : C, 57.20; $\mathrm{H}, 4.65$. Found: C, 57.42; H, 4.60.

1,6-Di-O-acetyl-2,3,4-tri-O-benzoyl- $\alpha$-Dmannopyranose (7). - A solution of mannose 6 $(15 \mathrm{~g}, 83.3 \mathrm{mmol})$ and chlorotriphenylmethane $(28 \mathrm{~g}, 100.5 \mathrm{mmol})$ in pyridine $(60 \mathrm{~mL})$ was stirred at $50^{\circ} \mathrm{C}$ for $32 \mathrm{~h}$ until TLC (4:1 EtOAc- $\mathrm{MeOH})$ indicated that the reaction was complete. The reaction mixture was cooled to $0{ }^{\circ} \mathrm{C}$, and $\mathrm{BzCl}(46.5 \mathrm{~mL}, 370 \mathrm{mmol})$ was then added over 30 min keeping the reaction temperature under $40^{\circ} \mathrm{C}$. After $24 \mathrm{~h}$, water $(300 \mathrm{~mL})$ was added to the reaction mixture and stirring was continued for $30 \mathrm{~min}$. The aq solution was extracted with $\mathrm{CH}_{2} \mathrm{Cl}_{2}$ $(3 \times 100 \mathrm{~mL})$, and the extract was washed sequentially with $\mathrm{HCl}(1 \mathrm{~N})$ and satd aq $\mathrm{NaHCO}_{3}$ solution, dried $\left(\mathrm{Na}_{2} \mathrm{SO}_{4}\right)$, and concentrated. Without further separation, the residue was redissolved with $\mathrm{CH}_{2} \mathrm{Cl}_{2}(50 \mathrm{~mL})$, $\mathrm{Ac}_{2} \mathrm{O}(50 \mathrm{~mL})$ and $\mathrm{AcOH}(30 \mathrm{~mL})$, and cooled to $10{ }^{\circ} \mathrm{C}$ in an ice bath. Concd $\mathrm{H}_{2} \mathrm{SO}_{4}(8.8$ $\mathrm{mL}$ ) was added dropwise over $20 \mathrm{~min}$, then held for $20 \mathrm{~h}$ at $\mathrm{rt}$. The reaction solution was poured into ice water $(400 \mathrm{~mL})$, stirred for an additional $15 \mathrm{~min}$, and extracted with $\mathrm{CHCl}_{3}$ $(3 \times 100 \mathrm{~mL})$. Combined extracts were washed with $10 \%$ aq $\mathrm{NaHCO}_{3}(3 \times 60 \mathrm{~mL})$, dried, and concentrated to give a syrup. Column chromatography with 4:1 petroleum ether-EtOAc as the eluent afforded compound 7 as a syrup (34.2 g, 71.3\%); $[\alpha]_{\mathrm{D}}+9.5^{\circ}\left(c 1.0, \mathrm{CHCl}_{3}\right) ;{ }^{1} \mathrm{H}$ NMR (400 MHz, $\left.\mathrm{CDCl}_{3}\right): \delta 8.11-7.28(\mathrm{~m}, 15$ $\mathrm{H}, 3 \mathrm{Ph} H), 6.36\left(\mathrm{~d}, 1 \mathrm{H}, J_{1,2} 2.0 \mathrm{~Hz}, \mathrm{H}-1\right)$, $6.01\left(\mathrm{t}, 1 \mathrm{H}, J_{3,4}=J_{4,5}=10.2 \mathrm{~Hz}, \mathrm{H}-4\right), 5.88$ $\left(\mathrm{dd}, 1 \mathrm{H}, J_{2,3} 3.2, J_{3,4} 10.2 \mathrm{~Hz}, \mathrm{H}-3\right), 5.70$ (dd, $\left.1 \mathrm{H}, J_{1,2} 2.0, J_{2,3} 3.2 \mathrm{~Hz}, \mathrm{H}-2\right), 4.34(\mathrm{~m}, 2 \mathrm{H}$, $\mathrm{H}-5,6 \mathrm{a}), 4.27$ (dd, $1 \mathrm{H}, J_{6 \mathrm{a}, 6 \mathrm{~b}} 13.4, J_{5,6 \mathrm{~b}} 4.2$ $\mathrm{Hz}, \mathrm{H}-6 \mathrm{~b}), 2.28,2.08$ (2 s, $\left.6 \mathrm{H}, 2 \mathrm{COCH}_{3}\right)$. Anal. Calcd for $\mathrm{C}_{31} \mathrm{H}_{28} \mathrm{O}_{11}$ : C, 64.58; H, 4.89. Found: C, 64.67; H, 4.94.

6-O-Acetyl-2,3,4-tri-O-benzoyl- $\alpha$-D-mannopyranose (8). - A solution of compound 7 (5 $\mathrm{g}, 8.67 \mathrm{mmol})$ and benzylamine $(4 \mathrm{~mL}, 36.6$ $\mathrm{mmol})$ in anhyd THF $(30 \mathrm{~mL})$ was stirred at rt for $24 \mathrm{~h}$ until TLC $(3: 1$ petroleum etherEtOAc) indicated that the reaction was complete. The solution was concentrated, and the crude product was purified by flash-column chromatography on silica gel $3: 1$ petroleum ether-EtOAc) to give compound $\mathbf{8}$ as a syrup 
$(4.0 \mathrm{~g}, 86.2 \%) ;[\alpha]_{\mathrm{D}}+16.8^{\circ}\left(c 1.0, \mathrm{CHCl}_{3}\right) ;{ }^{1} \mathrm{H}$ NMR (400 MHz, $\left.\mathrm{CDCl}_{3}\right): \delta 8.11-7.27(\mathrm{~m}, 15$ $\mathrm{H}, 3 \mathrm{PhH}), 5.98$ (m, $2 \mathrm{H}, \mathrm{H}-3,4), 5.71$ (dd, 1 $\left.\mathrm{H}, J_{1,2} 1.6, J_{2,3} 2.4 \mathrm{~Hz}, \mathrm{H}-2\right), 5.53\left(\mathrm{~d}, 1 \mathrm{H}, J_{1,2}\right.$ $1.6 \mathrm{~Hz}, \mathrm{H}-1), 4.55$ (m, $1 \mathrm{H}, \mathrm{H}-5), 4.33$ (m, 2 $\mathrm{H}, \mathrm{H}-6 \mathrm{a}, 6 \mathrm{~b}), 2.09$ (s, $\left.3 \mathrm{H}, \mathrm{COCH}_{3}\right)$. Anal. Calcd for $\mathrm{C}_{29} \mathrm{H}_{26} \mathrm{O}_{10}$ : C, 65.16; H, 4.90. Found: C, 65.36; H, 4.84.

6-O-Acetyl-2,3,4-tri-O-benzoyl- $\alpha$-D-mannopyranosyl trichloroacetimidate (9). - A mixture of 8 (4.0 g, $7.48 \mathrm{mmol}), \mathrm{CCl}_{3} \mathrm{CN}$ (2.1 mL, 20.9 $\mathrm{mmol})$, and 1,8-diazabicyclo[5,4,0]undecene (DBU) $(0.25 \mathrm{~mL}, 1.67 \mathrm{mmol})$ in dry $\mathrm{CH}_{2} \mathrm{Cl}_{2}$ (25 mL) was stirred under $\mathrm{N}_{2}$ for $2 \mathrm{~h}$ at $0{ }^{\circ} \mathrm{C}$ and then concentrated in vacuo. The residue was purified by flash chromatography (4:1 petroleum ether-EtOAc) to give 9 (4.47 g, $88.1 \%)$ as crystals; $m p 88-91{ }^{\circ} \mathrm{C} ;[\alpha]_{\mathrm{D}}+20.3^{\circ}$ (c 1.0, $\mathrm{CHCl}_{3}$ ); ${ }^{1} \mathrm{H}$ NMR (400 $\left.\mathrm{MHz}, \mathrm{CDCl}_{3}\right)$ : $\delta 8.87\left(\mathrm{~s}, 1 \mathrm{H}, \mathrm{OC}(\mathrm{NH}) \mathrm{CCI}_{3}\right), 8.12-7.27(\mathrm{~m}$, $15 \mathrm{H}, 3 \mathrm{Ph} H), 6.55$ (d, $\left.1 \mathrm{H}, J_{1,2} 2.0 \mathrm{~Hz}, \mathrm{H}-1\right)$, $6.06\left(\mathrm{t}, 1 \mathrm{H}, J_{3,4}=J_{4,5}=10.0 \mathrm{~Hz}, \mathrm{H}-4\right), 5.93$ $\left(\mathrm{dd}, 1 \mathrm{H}, J_{2,3} 3.2, J_{3,4} 10.0 \mathrm{~Hz}, \mathrm{H}-3\right), 5.90(\mathrm{dd}$, $\left.1 \mathrm{H}, J_{1,2} 2.0, J_{2,3} 3.2 \mathrm{~Hz}, \mathrm{H}-2\right), 4.49(\mathrm{~m}, 1 \mathrm{H}$, $\mathrm{H}-5), 4.34$ (m, $2 \mathrm{H}, \mathrm{H}-6 \mathrm{a}, 6 \mathrm{~b}), 2.07$ (s, 3 H, $\mathrm{COCH}_{3}$ ). Anal. Calcd for $\mathrm{C}_{31} \mathrm{H}_{26} \mathrm{Cl}_{3} \mathrm{NO}_{10}$ : C, 54.84; H, 3.86. Found: C, 54.72; H, 3.90.

Allyl 6-O-acetyl-2-O-benzoyl-3,4-di-O-ben$z y l-\alpha$-D-mannopyranoside (10). - A solution of $3(2.5 \mathrm{~g}, 4.56 \mathrm{mmol})$ and allyl alcohol $(0.6 \mathrm{~mL}$, $8.8 \mathrm{mmol})$ in dry $\mathrm{CH}_{2} \mathrm{Cl}_{2}(40 \mathrm{~mL})$ was stirred with dried molecular sieves $(4 \AA, 1 \mathrm{~g})$ under $\mathrm{N}_{2}$ for $15 \mathrm{~min}$, and then trimethylsilyl trifluoromethanesulfonate $(0.2 \mathrm{~mL}, 1.1 \mathrm{mmol})$ was added dropwise. After $1 \mathrm{~h}$, the reaction mixture was diluted with $\mathrm{CH}_{2} \mathrm{Cl}_{2}(50 \mathrm{~mL})$ and washed with satd aq $\mathrm{NaHCO}_{3}$ solution (15 $\mathrm{mL}$ ). The organic layer was dried and concentrated in vacuo. Purification of the residue by flash chromatography (3:1 petroleum etherEtOAc) gave 10 as a syrup $(2.19 \mathrm{~g}, 88 \%) ;[\alpha]_{\mathrm{D}}$ $+5.9^{\circ}\left(c\right.$ 1.0, $\left.\mathrm{CHCl}_{3}\right)$; ${ }^{1} \mathrm{H}$ NMR $(400 \mathrm{MHz}$, $\left.\mathrm{CDCl}_{3}\right): \delta 8.09-7.24(\mathrm{~m}, 15 \mathrm{H}, 3 \mathrm{PhH}), 5.88$ $\left(\mathrm{m}, 1 \mathrm{H}, \mathrm{CH}_{2} \mathrm{CH}=\mathrm{CH}_{2}\right), 5.63\left(\mathrm{dd}, J_{1,2} 2.0, J_{3,2}\right.$ $3.0 \mathrm{~Hz}, \mathrm{H}-2), 5.28\left(\mathrm{dd}, 1 \mathrm{H},{ }^{2} \mathrm{~J} 1.6,{ }^{3} J_{\text {trans }}=\right.$ $\left.17.2 \mathrm{~Hz}, \mathrm{CH}_{2} \mathrm{CH}=\mathrm{CH}_{2}\right), 5.21\left(\mathrm{dd}, 1 \mathrm{H},{ }^{2} \mathrm{~J} 1.6\right.$, $\left.{ }^{3} J_{\text {cis }} 10.4 \mathrm{~Hz}, \mathrm{CH}_{2} \mathrm{CH}=\mathrm{CH}_{2}\right), 4.98\left(\mathrm{~d}, J_{1,2} 2.0\right.$ $\mathrm{Hz}, \mathrm{H}-1), 4.88,4.59(\mathrm{ABq}, 2 \mathrm{H}, J 10.8 \mathrm{~Hz}$, $\left.\mathrm{PhCH}_{2}\right), 4.80,4.58\left(\mathrm{ABq}, J 11.2 \mathrm{~Hz}, \mathrm{PhCH}_{2}\right)$, 4.36 (m, $2 \mathrm{H}, \mathrm{H}-6 \mathrm{a}, 6 \mathrm{~b}), 4.20-3.90$ (m, $5 \mathrm{H}$, $\mathrm{H}-3,4,5, \mathrm{CH}_{2} \mathrm{CH}=\mathrm{CH}_{2}$ ), 2.07 (s, $3 \mathrm{H}, \mathrm{CO}-$
$\mathrm{CH}_{3}$ ). Anal. Calcd for $\mathrm{C}_{32} \mathrm{H}_{34} \mathrm{O}_{8}: \mathrm{C}, 70.31 ; \mathrm{H}$, 6.27. Found: C, 70.24; H, 6.31.

Allyl 2-O - benzoyl-3,4-di - O - benzyl - $\alpha$ - Dmannopyranoside (11). - A solution of $\mathbf{1 0}$ (1.6 g, $2.93 \mathrm{mmol})$ in $\mathrm{MeOH}(80 \mathrm{~mL})$ containing $0.5 \% \mathrm{HCl}$ was stirred at rt for $18 \mathrm{~h}$, neutralized with $\mathrm{Et}_{3} \mathrm{~N}$, and then evaporated to dryness. The residue was partitioned between water and $\mathrm{CH}_{2} \mathrm{Cl}_{2}$, and the organic layer was dried and concentrated to a syrup. Purification of the residue by flash chromatography (2:1 petroleum ether-EtOAc) gave $\mathbf{1 1}$ as a syrup $(1.45 \mathrm{~g}, 98.1 \%) ;[\alpha]_{\mathrm{D}}+9.2^{\circ}(c) 1.0$, $\left.\mathrm{CHCl}_{3}\right) ;{ }^{1} \mathrm{H}$ NMR $\left(400 \mathrm{MHz}, \mathrm{CDCl}_{3}\right): \delta$ $8.08-7.23(\mathrm{~m}, 15 \mathrm{H}, 3 \mathrm{PhH}), 5.88(\mathrm{~m}, 1 \mathrm{H}$, $\left.\mathrm{CH}_{2} \mathrm{CH}=\mathrm{CH}_{2}\right), 5.61\left(\mathrm{dd}, J_{1,2} 1.8, J_{2,3} 3.6 \mathrm{~Hz}\right.$, $\mathrm{H}-2), 5.28\left(\mathrm{dd}, 1 \mathrm{H},{ }^{2} J 1.6,{ }^{3} J_{\text {trans }} 17.2 \mathrm{~Hz}\right.$, $\left.\mathrm{CH}_{2} \mathrm{CH}=\mathrm{CH}_{2}\right), 5.21\left(\mathrm{dd}, 1 \mathrm{H},{ }^{2} \mathrm{~J} 1.6,{ }^{3} J_{\text {cis }} 10.4\right.$ $\left.\mathrm{Hz}, \mathrm{CH}_{2} \mathrm{CH}=\mathrm{CH}_{2}\right), 4.97$ (d, $\left.J_{1,2} 1.8 \mathrm{~Hz}, \mathrm{H}-1\right)$, $4.92,4.65\left(\mathrm{ABq}, 2 \mathrm{H}, J 10.8 \mathrm{~Hz}, \mathrm{PhCH}_{2}\right)$, $4.78,4.58\left(\mathrm{ABq}, 2 \mathrm{H}, J 11.2 \mathrm{~Hz}, \mathrm{PhCH}_{2}\right)$, 4.15-3.78 (m, $7 \mathrm{H}, \mathrm{H}-3,4,5,6 \mathrm{a}, 6 \mathrm{~b}$, $\mathrm{CH}_{2} \mathrm{CH}=\mathrm{CH}_{2}$ ). Anal. Calcd for $\mathrm{C}_{30} \mathrm{H}_{32} \mathrm{O}_{7}: \mathrm{C}$, 71.41; H, 6.39. Found: C, 71.04; H, 6.35.

Allyl 6-O-acetyl-2-O-benzoyl-3,4-di-O-benzyl- $\alpha$-D-mannopyranosyl- $(1 \rightarrow 6)-2-\mathrm{O}-$ benzoyl3,4-di-O-benzyl- $\alpha$-D-mannopyranoside (12). A solution of $11(0.37 \mathrm{~g}, 0.73 \mathrm{mmol})$ and trimethylsilyl trifluoromethanesulfonate (13 $\mu \mathrm{L}, 0.073 \mathrm{mmol})$ in dry $\mathrm{CH}_{2} \mathrm{Cl}_{2}(6 \mathrm{~mL})$ was stirred with dried molecular sieves $(4 \AA, 0.4 \mathrm{~g})$ under $\mathrm{N}_{2}$ for $15 \mathrm{~min}$, and then $5(0.65 \mathrm{~g}, 1.0$ $\mathrm{mmol})$ in $\mathrm{CH}_{2} \mathrm{Cl}_{2}(4 \mathrm{~mL})$ was added over 20 min at $\mathrm{rt}$. After $3 \mathrm{~h}$, the reaction mixture was diluted with $\mathrm{CH}_{2} \mathrm{Cl}_{2}(30 \mathrm{~mL})$ and washed with satd aq $\mathrm{NaHCO}_{3}$ solution $(5 \mathrm{~mL})$. The organic layer was dried and concentrated in vacuo. Purification of the residue by flash chromatography (2:1 petroleum etherEtOAc) gave 12 as a syrup $(0.63 \mathrm{~g}, 86.5 \%)$; $[\alpha]_{\mathrm{D}}+19.3^{\circ}\left(c 1.0, \mathrm{CHCl}_{3}\right) ;{ }^{1} \mathrm{H}$ NMR $(400$ $\left.\mathrm{MHz}, \mathrm{CDCl}_{3}\right): \delta 8.11-7.22(\mathrm{~m}, 30 \mathrm{H}, 6 \mathrm{PhH})$, $5.88\left(\mathrm{~m}, 1 \mathrm{H}, \mathrm{CH}_{2} \mathrm{CH}=\mathrm{CH}_{2}\right), 5.73(\mathrm{dd}, 1 \mathrm{H}$, $\left.J_{1,2} 1.6, J_{2,3} 3.2 \mathrm{~Hz}, \mathrm{H}-2\right), 5.67\left(\mathrm{dd}, 1 \mathrm{H}, J_{1^{\prime}, 2}\right.$ $\left.1.6, J_{2^{\prime}, 3^{\prime}} 3.2 \mathrm{~Hz}, \mathrm{H}-2^{\prime}\right), 5.28\left(\mathrm{dd}, 1 \mathrm{H},{ }^{2} J 1.6\right.$, $\left.{ }^{3} J_{\text {trans }} 17.2 \mathrm{~Hz}, \mathrm{CH}_{2} \mathrm{CH}=\mathrm{CH}_{2}\right), 5.21(\mathrm{dd}, 1 \mathrm{H}$, $\left.{ }^{2} J 1.6,{ }^{3} J_{\text {cis }}=10.4 \mathrm{~Hz}, \mathrm{CH}_{2} \mathrm{CH}=\mathrm{CH}_{2}\right), 5.08(\mathrm{~d}$, $\left.1 \mathrm{H}, J_{1,2} 1.6 \mathrm{~Hz}, \mathrm{H}-1\right), 4.97\left(\mathrm{~d}, 1 \mathrm{H}, J_{1^{\prime}, 2^{\prime}} 1.6\right.$ $\left.\mathrm{Hz}, \mathrm{H}-1^{\prime}\right), 4.90-4.45$ (m, $\left.8 \mathrm{H}, 4 \mathrm{PhCH}_{2}\right)$, 4.28-3.76 (m, $12 \mathrm{H}), 1.99\left(\mathrm{~s}, 3 \mathrm{H}, \mathrm{COCH}_{3}\right)$. Anal. Calcd for $\mathrm{C}_{59} \mathrm{H}_{60} \mathrm{O}_{14}: \mathrm{C}, 71.36 ; \mathrm{H}, 6.09$. Found: C, 71.45; H, 6.11. 
Allyl 2-O - benzoyl-3,4-di-O - benzyl - $\alpha$ - Dmannopyranosyl- $(1 \rightarrow 6)-2-\mathrm{O}-$ benzoyl-3,4-diO-benzyl- $\alpha$-D-mannopyranoside (13). - A solution of 12 (0.55 g, $0.55 \mathrm{mmol})$ in $\mathrm{MeOH}(25$ $\mathrm{mL}$ ) containing $0.5 \% \mathrm{HCl}$ was stirred at $\mathrm{rt}$ for $14 \mathrm{~h}$. The mixture was carefully neutralized with $\mathrm{Et}_{3} \mathrm{~N}$, and then evaporated to dryness. The residue was partitioned between water and $\mathrm{CH}_{2} \mathrm{Cl}_{2}$, then the organic layer was dried and evaporated to a syrup. Purification of the residue by flash chromatography (2:1 petroleum ether-EtOAc) gave $\mathbf{1 3}$ as a syrup $(0.50 \mathrm{~g}, 95.6 \%) ;[\alpha]_{\mathrm{D}}+13.0^{\circ}\left(c \mathrm{1.5}, \mathrm{CHCl}_{3}\right)$; ${ }^{1} \mathrm{H}$ NMR $\left(400 \mathrm{MHz}, \mathrm{CDCl}_{3}\right): \delta 8.08-7.15(\mathrm{~m}$, $30 \mathrm{H}, 6 \mathrm{Ph} H), 5.87\left(\mathrm{~m}, 1 \mathrm{H}, \mathrm{CH}_{2} \mathrm{CH}=\mathrm{CH}_{2}\right)$, $5.72\left(\mathrm{dd}, 1 \mathrm{H}, J_{1,2} 1.6, J_{2,3} 3.2 \mathrm{~Hz}, \mathrm{H}-2\right), 5.66$ $\left(\mathrm{dd}, 1 \mathrm{H}, J_{1^{\prime}, 2^{\prime}} 1.6, J_{2^{\prime}, 3^{\prime}} 3.2 \mathrm{~Hz}, \mathrm{H}-2^{\prime}\right), 5.28$ (dd, $\left.1 \mathrm{H},{ }^{2} \mathrm{~J} 1.6,{ }^{3} J_{\text {trans }} 17.2 \mathrm{~Hz}, \mathrm{CH}_{2} \mathrm{CH}=\mathrm{CH}_{2}\right), 5.20$ $\left(\mathrm{dd}, 1 \mathrm{H},{ }^{2} \mathrm{~J} 1.6,{ }^{3} J_{\text {cis }} 10.4 \mathrm{~Hz}, \mathrm{CH}_{2} \mathrm{CH}=\mathrm{CH}_{2}\right.$ ), $5.07\left(\mathrm{~d}, 1 \mathrm{H}, J_{1,2} 1.6 \mathrm{~Hz}, \mathrm{H}-1\right), 4.96(\mathrm{~d}, 1 \mathrm{H}$, $\left.J_{1^{\prime}, 2^{\prime}} 1.6 \mathrm{~Hz}, \mathrm{H}-1^{\prime}\right), 4.90-4.45$ (m, $8 \mathrm{H}, 4$ $\left.\mathrm{PhCH}_{2}\right), 4.14-3.71(\mathrm{~m}, 12 \mathrm{H})$. Anal. Calcd for $\mathrm{C}_{57} \mathrm{H}_{58} \mathrm{O}_{13}: \mathrm{C}, 71.98 ; \mathrm{H}, 6.15$. Found: C, 71.87; $\mathrm{H}, 6.18$.

Allyl 2-O - acetyl-3,4,6-tri-O - benzyl- $\alpha$ - Dmannopyranosyl- $(1 \rightarrow 6)-2-\mathrm{O}-$ benzoyl-3,4-di$\mathrm{O}$ - benzyl- $\alpha$ - D - mannopyranosyl - $(1 \rightarrow 6)$ - 2-Obenzoyl-3,4-di-O-benzyl- $\alpha$-D-mannopyranoside (14). - A solution of $13(0.25 \mathrm{~g}, 0.26 \mathrm{mmol})$ and trimethylsilyl trifluoromethanesulfonate (5 $\mu \mathrm{L}, 0.028 \mathrm{mmol})$ in dry $\mathrm{CH}_{2} \mathrm{Cl}_{2}(20 \mathrm{~mL})$ was stirred with dried molecular sieves ( $4 \AA, 1$ g) under $\mathrm{N}_{2}$ for $15 \mathrm{~min}$, and then 2-O-acetyl3,4,6-tri- $O$-benzyl- $\alpha$-D-mannopyranosyl trichloroacetimidate (12) $(0.25 \mathrm{~g}, 0.39 \mathrm{mmol})$ in dry $\mathrm{CH}_{2} \mathrm{Cl}_{2}(10 \mathrm{~mL})$ was added over $30 \mathrm{~min}$ at rt. After $3 \mathrm{~h}$ the reaction mixture was diluted with $\mathrm{CH}_{2} \mathrm{Cl}_{2}(20 \mathrm{~mL})$ and washed with satd aq $\mathrm{NaHCO}_{3}$ solution $(8 \mathrm{~mL})$. The organic layer was dried and concentrated in vacuo. Purification of the residue by column chromatography (2:1 petroleum ether-EtOAc) gave $\mathbf{1 4}$ as a syrup $(0.32 \mathrm{~g}, 85.7 \%) ;[\alpha]_{\mathrm{D}}+30.7^{\circ}$ (c 1.0, $\left.\mathrm{CHCl}_{3}\right) ;{ }^{1} \mathrm{H}$ NMR (400 $\left.\mathrm{MHz}, \mathrm{CDCl}_{3}\right): \delta$ 8.12-7.13 (m, $45 \mathrm{H}, 9 \mathrm{PhH}), 5.87(\mathrm{~m}, 1 \mathrm{H}$, $\left.\mathrm{CH}_{2} \mathrm{CH}=\mathrm{CH}_{2}\right), 5.78\left(\mathrm{dd}, 1 \mathrm{H}, J_{1,2} 1.6, J_{2,3} 3.2\right.$ $\mathrm{Hz}, \mathrm{H}-2), 5.67$ (dd, $1 \mathrm{H}, J_{1^{\prime}, 2^{\prime}} 1.6, J_{2^{\prime}, 3^{\prime}} 3.2 \mathrm{~Hz}$, $\left.\mathrm{H}-2^{\prime}\right), 5.52\left(\mathrm{dd}, 1 \mathrm{H}, J_{1^{\prime \prime}, 2^{\prime \prime}} 1.6, J_{2^{\prime \prime}, 3^{\prime \prime}} 3.2 \mathrm{~Hz}\right.$, $\left.\mathrm{H}-2^{\prime \prime}\right), 5.28\left(\mathrm{dd}, 1 \mathrm{H},{ }^{2} J 1.6,{ }^{3} J_{\text {trans }} 17.2 \mathrm{~Hz}\right.$, $\left.\mathrm{CH}_{2} \mathrm{CH}=\mathrm{CH}_{2}\right), 5.19\left(\mathrm{dd}, 1 \mathrm{H},{ }^{2} J 1.6,{ }^{3} J_{\text {cis }} 10.4\right.$ $\left.\mathrm{Hz}, \mathrm{CH}_{2} \mathrm{CH}=\mathrm{CH}_{2}\right), 5.07$ (d, $1 \mathrm{H}, J_{1,2} 1.6 \mathrm{~Hz}$,
H-1), 4.98 (d, $\left.1 \mathrm{H}, J_{1^{\prime}, 2^{\prime}} 1.6 \mathrm{~Hz}, \mathrm{H}-1^{\prime}\right), 4.96$ (d, $\left.1 \mathrm{H}, J_{1^{\prime \prime}, 2^{\prime \prime}} 1.6 \mathrm{~Hz}, \mathrm{H}-1^{\prime \prime}\right), 4.87-4.37(\mathrm{~m}, 14 \mathrm{H}$, $\left.7 \mathrm{PhCH}_{2}\right), 4.14-3.61(\mathrm{~m}, 17 \mathrm{H}), 2.14(\mathrm{~s}, 3 \mathrm{H}$, $\mathrm{COCH}_{3}$ ). Anal. Calcd for $\mathrm{C}_{86} \mathrm{H}_{88} \mathrm{O}_{19}: \mathrm{C}, 72.46$; H, 6.22. Found: C, 72.53; H, 6.17.

Allyl 3,4,6-tri-O-benzyl- $\alpha$-D - mannopyranosyl- $(1 \rightarrow 6)-3,4-$ di - O - benzyl- $\alpha-\mathrm{D}-$ mannopy ranosyl- $(1 \rightarrow 6)-3,4-d i-\mathrm{O}-$ benzyl- $\alpha-\mathrm{D}-$ mannopyranoside (15). - A solution of $14(0.45 \mathrm{~g}$, $0.32 \mathrm{mmol})$ in $\mathrm{MeOH}(50 \mathrm{~mL})$ saturated with dry $\mathrm{NH}_{3}$ was stirred at rt for $72 \mathrm{~h}$, until TLC (1:1.5 petroleum ether-EtOAc) indicated that the reaction was complete. The solution was concentrated to afford compound $15(0.36 \mathrm{~g}$, $97 \%$ ) as a syrup; $[\alpha]_{\mathrm{D}}+21.4^{\circ}\left(c 1.0, \mathrm{CHCl}_{3}\right)$; ${ }^{1} \mathrm{H}$ NMR (400 MHz, $\left.\mathrm{CDCl}_{3}\right): \delta 7.81-7.15(\mathrm{~m}$, $35 \mathrm{H}, 7 \mathrm{PhH}), 5.85\left(\mathrm{~m}, 1 \mathrm{H}, \mathrm{CH}_{2} \mathrm{CH}=\mathrm{CH}_{2}\right)$, $5.23\left(\mathrm{dd}, 1 \mathrm{H},{ }^{2} J 1.6,{ }^{3} J_{\text {trans }} 17.2 \mathrm{~Hz}\right.$, $\left.\mathrm{CH}_{2} \mathrm{CH}=\mathrm{CH}_{2}\right), 5.12\left(\mathrm{dd}, 1 \mathrm{H},{ }^{2} \mathrm{~J} 1.6,{ }^{3} J_{\text {cis }} 10.4\right.$ $\left.\mathrm{Hz}, \mathrm{CH}_{2} \mathrm{CH}=\mathrm{CH}_{2}\right), 4.96\left(1 \mathrm{H}, J_{1,2} 2.0 \mathrm{~Hz}\right.$, $\mathrm{H}-1), 4.90$ (d, $\left.1 \mathrm{H}, J_{1^{\prime}, 2}, 2.0 \mathrm{~Hz}, \mathrm{H}_{-1} 1^{\prime}\right), 4.85$ (d, $\left.1 \mathrm{H}, J_{1^{\prime \prime}, 2^{\prime \prime}} 2.0 \mathrm{~Hz}, \mathrm{H}-1^{\prime \prime}\right), 4.82-4.46(\mathrm{~m}, 14 \mathrm{H}$, $\left.7 \mathrm{PhCH}_{2}\right), 4.07-3.64(\mathrm{~m}, 20 \mathrm{H})$. Anal. Calcd for $\mathrm{C}_{70} \mathrm{H}_{78} \mathrm{O}_{16}: \mathrm{C}, 71.53 ; \mathrm{H}, 6.69$. Found: $\mathrm{C}$, 71.61; H, 6.73.

Allyl 6-O-acetyl-2,3,4-tri-O-benzoyl- $\alpha-\mathrm{D}-$ mannopyranosyl-( $1 \rightarrow 2)-3,4,6$ - tri-O-benzyl- $\alpha$ D-mannopyranosyl- $(1 \rightarrow 6)-[(6-\mathrm{O}$-acetyl-2,3,4tri-O-benzoyl- $\alpha$-D-mannopyranosyl)- $(1 \rightarrow 2)]$ (3,4-di-O-benzyl- $\alpha$-D-mannopyranosyl)- $(1 \rightarrow 6)$ [(6-O-acetyl-2,3,4-tri-O-benzoyl- $\alpha$-D-mannopyranosyl) - $(1 \rightarrow 2)]-3,4-d i-\mathrm{O}-$ benzyl - $\alpha$ - Dmannopyranoside (16). - A solution of 15 (0.13 g, $0.11 \mathrm{mmol})$ and trimethylsilyl trifluoromethanesulfonate ( $4 \mu \mathrm{L}, 0.022 \mathrm{mmol}$ ) in dry $\mathrm{CH}_{2} \mathrm{Cl}_{2}(15 \mathrm{~mL})$ was stirred with dried molecular sieves $(4 \AA, 0.4 \mathrm{~g})$ under $\mathrm{N}_{2}$ for 15 min, and then 6- $O$-acetyl-2,3,4-tri- $O$-benzoyl$\alpha$-D-mannopyranosyl trichloroacetimidate (9) (0.45 g, $0.66 \mathrm{mmol})$ in dry $\mathrm{CH}_{2} \mathrm{Cl}_{2}(10 \mathrm{~mL})$ was added dropwise for $30 \mathrm{~min}$ at $\mathrm{rt}$. After $3 \mathrm{~h}$ the reaction mixture was diluted with $\mathrm{CH}_{2} \mathrm{Cl}_{2}(30 \mathrm{~mL})$ and washed with satd aq $\mathrm{NaHCO}_{3}$ solution $(5 \mathrm{~mL})$. The organic layer was dried and concentrated in vacuo. Purification of the residue by column chromatography (2:1 petroleum ether-EtOAc) gave $\mathbf{1 6}$ as a $\operatorname{syrup}(0.23 \mathrm{~g}, 75.8 \%) ;[\alpha]_{\mathrm{D}}+13.3^{\circ}$ (c 1.0, $\left.\mathrm{CHCl}_{3}\right) ;{ }^{1} \mathrm{H}$ NMR $\left(400 \mathrm{MHz}, \mathrm{CDCl}_{3}\right): \delta$ 8.03-7.14 (m, $80 \mathrm{H}, 16 \mathrm{PhH}), 6.02-5.92(\mathrm{~m}$, $10 \mathrm{H}, 2_{\mathrm{B}}, 2_{\mathrm{D}}, 2_{\mathrm{F}}, 3_{\mathrm{B}}, 3_{\mathrm{D}}, 3_{\mathrm{F}}, 4_{\mathrm{B}}, 4_{\mathrm{D}}, 4_{\mathrm{F}}$, 
$\left.\mathrm{CH}_{2} \mathrm{CH}=\mathrm{CH}_{2}\right), 5.56\left(\mathrm{~d}, 1 \mathrm{H}, J_{2,1} 1.1 \mathrm{~Hz}, \mathrm{H}_{\mathrm{B}}-\right.$ 1), $5.46\left(\mathrm{~d}, 1 \mathrm{H}, J_{2,1} 1.1 \mathrm{~Hz}, \mathrm{H}_{\mathrm{D}}-1\right), 5.40(\mathrm{~d}, 1$ $\left.\mathrm{H}, J_{2,1} 1.1 \mathrm{~Hz}, \mathrm{H}_{\mathrm{F}}-1\right), 5.28\left(\mathrm{dd}, 1 \mathrm{H},{ }^{2} J 1.6\right.$, $\left.{ }^{3} J_{\text {trans }} 17.2 \mathrm{~Hz}, \mathrm{CH}_{2} \mathrm{CH}=\mathrm{CH}_{2}\right), 5.21(\mathrm{~d}, 1 \mathrm{H}$, $\left.J_{2.1} 1.1 \mathrm{~Hz}, \mathrm{H}_{\mathrm{A}}-1\right), 5.18\left(\mathrm{dd}, 1 \mathrm{H},{ }^{2} J 1.6,{ }^{3} J_{\text {cis }}\right.$ $\left.10.4 \mathrm{~Hz}, \mathrm{CH}_{2} \mathrm{CH}=\mathrm{CH}_{2}\right), 5.14\left(\mathrm{~d}, 1 \mathrm{H}, J_{2,1} 1.1\right.$ $\left.\mathrm{Hz}, \mathrm{H}_{\mathrm{C}}-1\right), 4.97\left(\mathrm{~d}, 1 \mathrm{H}, J_{2,1} 1.1 \mathrm{~Hz}, \mathrm{H}_{\mathrm{E}}-1\right)$, $2.07,1.99,1.97\left(3 \mathrm{~s}, 9 \mathrm{H}, 3 \mathrm{COCH}_{3}\right) ;{ }^{13} \mathrm{C}$ NMR $\left(400 \mathrm{MHz}, \mathrm{CDCI}_{3}\right): \delta$ 170.5-170.4 $\left(\mathrm{CH}_{3} \mathrm{CO}\right), 165.7-164.8$ (9 PhCO), 138.8-137.7 (7 $\left.\mathrm{PhCH}_{2}, \mathrm{C}-1\right), 133.4-132.7$ (7 PhCO, C-1, $\mathrm{CH}_{2}=\mathrm{CH}-\mathrm{CH}_{2}$ ), 129.9-127.2 (aromatic C), $117.5\left(\mathrm{CH}_{2}=\mathrm{CH}-\mathrm{CH}_{2}\right), 99.6,99.4,99.3,99.2$, 99.1, $98.3\left(\mathrm{C}-1_{\mathrm{A}}, 1_{\mathrm{B}}, 1_{\mathrm{C}}, 1_{\mathrm{D}}, 1_{\mathrm{E}}, 1_{\mathrm{F}}\right), 80.2,80.0$, $79.0\left(\mathrm{C}-2_{\mathrm{B}}, \quad 2_{\mathrm{C}}, 2_{\mathrm{E}}\right), 67.9 \quad\left(\mathrm{CH}_{2}=\mathrm{CH}-\mathrm{CH}_{2}\right)$, 20.61, 20.58, 20.53 (3 $\left.\mathrm{CH}_{3} \mathrm{CO}\right)$; Anal. Calcd for $\mathrm{C}_{157} \mathrm{H}_{150} \mathrm{O}_{43}: \mathrm{C}, 69.20$; $\mathrm{H}, 5.55$. Found: $\mathrm{C}$, 69.14; H, 5.59.

$\alpha$-D - Mannopyranosyl- $(1 \rightarrow 2)-\alpha-\mathrm{D}$ - mannopyranosyl - $(1 \rightarrow 6)$ - [ $\alpha$ - D - mannopyranosyl$(1 \rightarrow 2)]-\alpha-D$ - mannopyranosyl - $(1 \rightarrow 6)-[(\alpha-\mathrm{D}-$ mannopyranose $)$ - $(1 \rightarrow 2)]$ - D - mannopyranose (1).-A mixture of compound $\mathbf{1 6}(0.1 \mathrm{~g}, 0.037$ $\mathrm{mmol})$ and $\mathrm{PdCl}_{2}(5 \mathrm{mg}, 0.028 \mathrm{mmol})$ in dry $\mathrm{MeOH}(10 \mathrm{~mL})$ was stirred vigorously for $8 \mathrm{~h}$ at $\mathrm{rt}$, then filtered through Celite. The filtrate was concentrated to dryness, redissolved in $\mathrm{MeOH}(20 \mathrm{~mL})$ saturated with dry $\mathrm{NH}_{3}$, and stirred at $\mathrm{rt}$ for $72 \mathrm{~h}$. After concentrating again, the residue was treated with $20 \mathrm{mg}$ of $20 \% \mathrm{Pd}(\mathrm{OH})_{2} / \mathrm{C}$ in $\mathrm{MeOH}(20 \mathrm{~mL})$ (caution: extreme fire hazard!) at $\mathrm{rt}$ under $\mathrm{H}_{2}$ for $24 \mathrm{~h}$. The catalyst was removed by filtration through a Celite pad, and washed twice with $\mathrm{MeOH}(30 \mathrm{~mL})$. The combined filtrate was concentrated to a residue which was chromatographed $(\mathrm{MeOH})$ on a column of Sep- hadex LH-20 to afford $1(0.022 \mathrm{~g}, 61.8 \%)$ as an amorphous mass; $[\alpha]_{\mathrm{D}}+1.4^{\circ}(c) 0.1$, $\mathrm{MeOH})$; ESMS for $\mathrm{C}_{36} \mathrm{H}_{62} \mathrm{O}_{31}$ (990.87): 989.5 $[\mathrm{M}-\mathrm{H}]$.

\section{Acknowledgements}

This work was supported by Chinese Academy of Sciences (KJ952 $\mathrm{J}_{1} 510$ and KIPRCEES9904) and The National Natural Science Foundation of China (59973026 and 29905004). Mr Linsen Heng is a visiting scholar from Da Xian Normal College, Department of Chemistry, Si Chuan, People's Republic of China.

\section{References}

[1] Lampen, J. O.; van Leeuwenhoek, A. J. Microbiol. Serol. 1968, 34, 1-18.

[2] Jiménez-Barbero, J.; Bernabé, M.; Leal, J. A.; Prieto, A.; Gómez-Miranda, B. Carbohydr. Res. 1993, 250, 289-299.

[3] Ning, J.; Kong, F. Tetrahedron Lett. 1999, 40, 13571360.

[4] Rachaman, E. S.; Eby, R.; Schuerch, C. Carbohydr. Res. 1978, 67, 147-161.

[5] Wilson, J. D.; Durham, N. C. U.S. Patent 4,921,950, 1999.

[6] Lemieux, R. U.; Howard, J. Methods Carbohydr. Chem. 1963, 2, 400-402.

[7] Barker, G. R. Methods Carbohydr. Chem. 1963, 2, 168 171.

[8] Helferich, B.; Schmitz-Hillebrecht, E. Ber. Dtsch. Chem. Ges. 1933, 66, 378-385.

[9] Schmidt, R. R.; Toepfer, A. Tetrahedron Lett. 1991, 32, 3353-3356.

[10] (a) Yamazaki, F.; Sato, S.; Nukada, T.; Tto, T.; Ogawa, T. Carbohydr. Res. 1990, 201, 31-50. (b) Paulsen, H.; Helpap, B. Carbohydr. Res. 1991, 216, 289-313. 